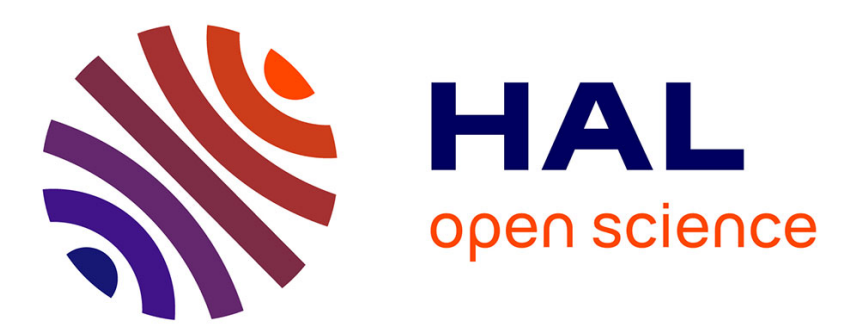

\title{
A study of the insulating spin-glass Eu0.44Sr0.56S by low temperature thermal conductivity measurements
}

\author{
C. Arzoumanian, A.M. de Goër, B. Salce, F. Holtzberg
}

\section{To cite this version:}

C. Arzoumanian, A.M. de Goër, B. Salce, F. Holtzberg. A study of the insulating spin-glass Eu0.44Sr0.56S by low temperature thermal conductivity measurements. Journal de Physique Lettres, 1983, 44 (1), pp.39-45. 10.1051/jphyslet:0198300440103900 . jpa-00232140

HAL Id: jpa-00232140

https://hal.science/jpa-00232140

Submitted on 1 Jan 1983

HAL is a multi-disciplinary open access archive for the deposit and dissemination of scientific research documents, whether they are published or not. The documents may come from teaching and research institutions in France or abroad, or from public or private research centers.
L'archive ouverte pluridisciplinaire $\mathbf{H A L}$, est destinée au dépôt et à la diffusion de documents scientifiques de niveau recherche, publiés ou non, émanant des établissements d'enseignement et de recherche français ou étrangers, des laboratoires publics ou privés. 
Classification

Physics Abstracts

$66.70-75.40$

\title{
A study of the insulating spin-glass $\mathrm{Eu}_{0.44} \mathrm{Sr}_{0.56} \mathrm{~S}$ by low temperature thermal conductivity measurements
}

\author{
C. Arzoumanian, A. M. De Goër, B. Salce \\ Laboratoire de Cryophysique, Service des Basses Températures, Centre d'Etudes Nucléaires, 85X, \\ 38041 Grenoble Cedex, France
}

and F. Holtzberg

I.B.M., Yorktown Heights, NY 10598, U.S.A.

(Reçu le 27 septembre 1982, accepté le 10 novembre 1982)

\begin{abstract}
Résumé. - Nous avons effectué des mesures de conductivité thermique sur un monocristal du verre de spin isolant $\mathrm{Eu}_{0,44} \mathrm{Sr}_{0,56} \mathrm{~S}$ entre $60 \mathrm{mK}$ et $70 \mathrm{~K}$. La conductivité thermique $K$ varie en $T^{2}$ aux températures inférieures à $3 \mathrm{~K}$ et nous n'avons observé aucune anomalie à $T_{\mathrm{g}}=1,8 \mathrm{~K}$ (température de gel déduite des mesures de susceptibilité magnétique alternative à $17 \mathrm{~Hz}$ ). Nous avons également étudié l'effet de l'application d'un champ magnétique de 7 teslas au-dessous de 1,2 $\mathrm{K}$ et une augmentation de $K$ d'environ $50 \%$ a été observée; ceci nous permet de conclure à une diffusion des phonons par des excitations magnétiques de basse énergie liées au désordre de la phase verre de spin. Une mesure en fonction du champ à température constante bien au-dessous de $T_{\mathrm{g}}$ a montré que l'augmentation de $K$ était saturée dès 2 teslas. Une analyse phénoménologique des courbes $K(T)$ a été faite $(T<1 \mathrm{~K})$ en supposant que l'effet des excitations magnétiques est analogue à celui des " systèmes à deux niveaux » (TLS) considérés dans le cas des verres de structure.
\end{abstract}

\begin{abstract}
Thermal conductivity measurements have been carried out on a single crystal of the insulating spin glass $\mathrm{Eu}_{0.44} \mathrm{Sr}_{0.56} \mathrm{~S}$ from $60 \mathrm{mK}$ to $70 \mathrm{~K}$. The thermal conductivity $K$ was found to vary as $T^{2}$ below $3 \mathrm{~K}$ and no anomaly has been observed at $T_{\mathrm{g}}=1.8 \mathrm{~K}$ (the value of the freezing temperature deduced from a-c magnetic susceptibility measurements at $17 \mathrm{~Hz}$ ). The effect of a magnetic field of 7 teslas is to increase the conductivity by about $50 \%$ below $1.2 \mathrm{~K}$, so that it can be inferred that phonon scattering by low lying magnetic excitations related to the spin glass phase disorder is present. From a measurement at a constant temperature well below $T_{\mathrm{g}}$ and as a function of field, it was found that the increase of $K$ saturates above 2 teslas. A phenomenological analysis of the $K(T)$ curves $(T<1 \mathrm{~K})$ has been made within the hypothesis that the effect of the magnetic excitations is analogous to that of the " two level systems " (TLS) considered in the case of structural glasses.
\end{abstract}

1. Introduction. - The system $\mathrm{Eu}_{x} \mathrm{Sr}_{1-x} \mathrm{~S}$ contains the first insulating material which was shown to display a magnetic field behaviour similar to that found in metallic spin glasses [1, 2]. The phase diagram (transition temperature as a function of Eu concentration) was established 
from different experiments, mostly on powder samples, and the so-called spin glass phase was observed in the concentration range $0.13<x<0.65$ [3]. For $x>0.65$ the system is ferromagnetic as is pure $\mathrm{EuS}$. EuS $\left(T_{\mathrm{c}}=16.7 \mathrm{~K}\right)$ has the $\mathrm{NaCl}$ f.c.c. structure with ferromagnetic exchange interactions between nearest neighbours and antiferromagnetic interactions between next nearest neighbours. In $\mathrm{Eu}_{x} \mathrm{Sr}_{1-x} \mathrm{~S}$, the $\mathrm{Eu}$ and $\mathrm{Sr}$ ions are randomly distributed on the sites of the same f.c.c. sublattice and the competition between the short range interactions of opposite sign gives the frustration effect which leads to the possibility of a spin glass state [4].

The absence of conduction electrons in $\mathrm{Eu}_{x} \mathrm{Sr}_{1-x} \mathrm{~S}$ is advantageous in the sense that some techniques used to study the spin glass phase give more direct evidence of characteristic properties than for metallic systems. This is the case with EPR experiments [5] and measurements of low temperature specific heat [6]. The evidence of a contribution to the specific heat which is proportional to the temperature (and which is sensitive to magnetic field) suggests the presence of low energy magnetic excitations with a constant density of states, by analogy with observations in structural glasses [7]. Therefore it is interesting to look for other characteristic properties of glasses at low temperatures [8], and their sensitivity to applied magnetic fields. In this letter, we present the first measurements of the thermal conductivity of an $\mathrm{Eu}_{0.44} \mathrm{Sr}_{0.56} \mathrm{~S}$ single crystal in the temperature range $60 \mathrm{mK}$ to $70 \mathrm{~K}$, and measurements with applied magnetic fields up to 7 teslas below $1 \mathrm{~K}$. Samples with the same concentration $x=0.44$ have been studied previously by a.-c. susceptibility measurements which have given a freezing temperature $T_{\mathrm{g}}=1.8 \mathrm{~K}$ at $17 \mathrm{~Hz}$ [9].

2. Experiments and results. - The single crystal used in the present work has a length of $5.5 \mathrm{~mm}$ and a cross section approximately $1 \mathrm{~mm}^{2}$.

The thermal conductivity was measured using the standard steady state longitudinal heat flow method. The thermometers were Speer carbon resistors and two different pairs were used above and below $1.2 \mathrm{~K}$. The low-temperature range was obtained with a dilution refrigerator and the magnetic field up to 7 teslas with a superconducting coil. The magnetic field was applied parallel to the heat flow. The magnetoresistance of the thermometers has been measured $(|\Delta R / R| \leqslant 15 \%$ below $1.2 \mathrm{~K}$ ) and taken into account to obtain the results with applied field. The accuracy of the calibration is about $1 \%$. The largest error on the absolute values of the thermal conductivity is about $10 \%$ and is due to the poor knowledge of the geometrical factor $l / S$ for such a small sample. This systematic error is not to be considered when the results with applied field and in zero field are compared and the estimation of the other sources of error leads to a relative accuracy of $3 \%$.

The results of the measurements in zero field from $50 \mathrm{mK}$ to $70 \mathrm{~K}$ are shown in figure 1 . The general shape is typical of an impure insulating crystal, with a broad maximum around $20 \mathrm{~K}$. There is no anomaly at the freezing temperature $T_{\mathrm{g}}$. The solid line in figure 1 corresponds to the limiting $T^{3}$ law (boundary scattering) estimated from the dimensions of the sample. In fact, the variation of the thermal conductivity is less than $T^{3}$ and a $T^{2}$ law is nearly followed below $3 \mathrm{~K}$. This temperature dependence is illustrated by the $K / T^{2}$ plot in the insert of figure 2 for the temperature range below $1 \mathrm{~K}$.

Two sets of experiments have been carried out with applied magnetic field. First a high field ( $H=7$ teslas) has been applied at $1.2 \mathrm{~K}$ and the thermal conductivity measured between $80 \mathrm{mK}$ and $1.2 \mathrm{~K}$. The results are shown in figure 2 and it is seen that the conductivity is increased by a factor of about $50 \%$ compared to its zero-field values, and that it still varies as $T^{2}$ (see the insert of figure 2). Second, a measurement at constant temperature $T=0.288 \mathrm{~K}$ was made as a function of decreasing magnetic field from 7 teslas (again applied at $1.2 \mathrm{~K}$ ) to zero. This temperature was chosen as the magnetoresistance of the thermometer was especially low $(|\Delta R / R| \leqslant 3 \%$ in the whole field range). The results are shown in figure 3 and it is seen that the field effect is saturated above 2 teslas. At the end of both experiments, the magnetic field was suppressed and the conductivity remeasured : the same values as found in the previous zero-field run were obtained within experimental accuracy so that no memory effect was detectable. 


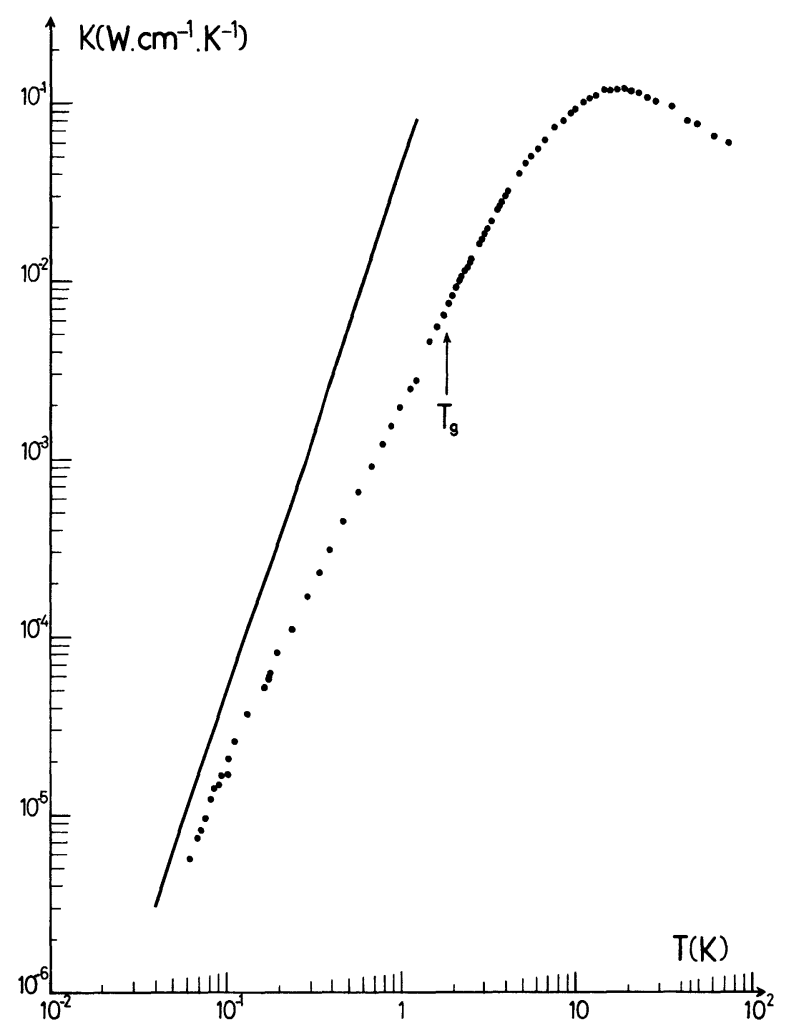

Fig. 1. - Zero field thermal conductivity of an $\mathrm{Eu}_{0.44} \mathrm{Sr}_{0.56} \mathrm{~S}$ single crystal as a function of temperature. The solid line is the calculated $T^{3}$ law (boundary scattering limit) for this sample.

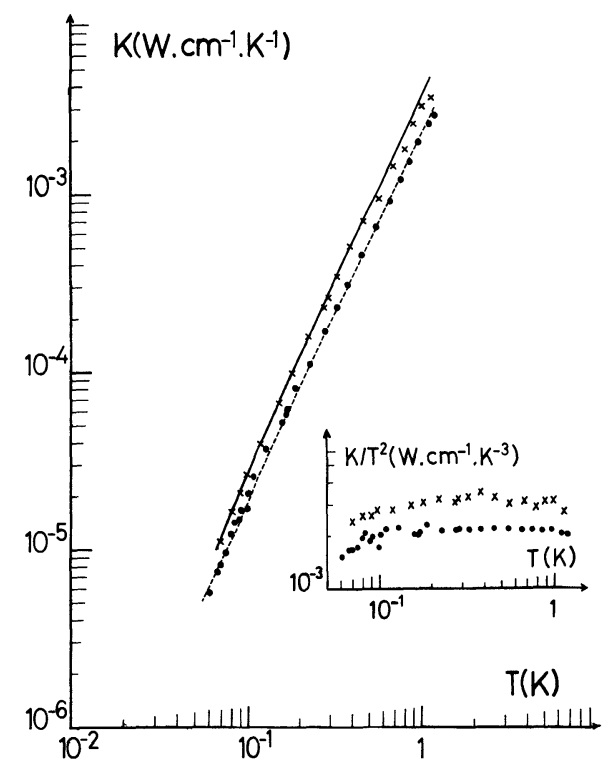

Fig. 2. - Thermal conductivity as a function of temperature for zero magnetic field; $x$ with an applied field of 7 teslas. The dashed and solid lines are calculated curves (see text). The insert shows the ratio $K / T^{2}$ as a function of temperature (the symbols are the same as above). 


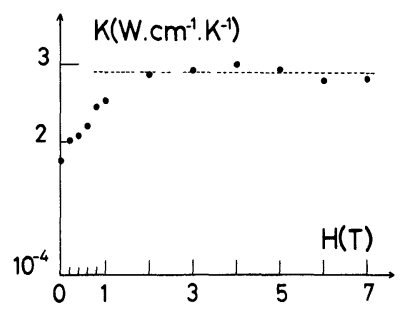

Fig. 3. - Thermal conductivity as a function of magnetic field at $T=0.288 \mathrm{~K}$. (The dashed line is a guide to the eye.)

3. Discussion. - The results in the temperature range $T<1 \mathrm{~K}$ can be qualitatively explained by the presence of low energy excitations already suggested by the results of specific heat measurements [6] as noted in the introduction. The phonons are resonantly scattered by such entities analogous to the TLS in structural glasses which involve tunnelling process; it is well known that a constant density of states $n_{0}$ leads to a thermal conductivity varying as $\sim \alpha T^{2}$ (if this scattering is predominant), where the constant $\alpha$ is inversely proportional to $n_{0} \bar{M}^{2}, \bar{M}$ being a mean coupling constant [8]. In the present case, the « TLS » are clearly related to the spin disorder as both the specific heat and the thermal conductivity are sensitive to applied magnetic field.

The results of these two experiments are coherent and the effect of large magnetic field is apparently to decrease the density $n_{0}$ of the «TLS » : the specific heat is actually decreased by the field and the thermal conductivity is increased. More quantitative comparison is not possible as the concentrations of the samples are slightly different $(x=0.4$ and $x=0.54)$ and, as the largest magnetic field used in the specific heat work is 1 tesla [6], the saturation observed in the present work (Fig. 3) has not been reached. We note that some phonon scattering is still present in large magnetic fields, as the $T^{3}$ law for boundary scattering alone is not recovered (Fig. 2). There is no anomaly at $T_{\mathrm{g}}$ on the zero field thermal conductivity curve (see Fig. 1) and preliminary measurements have shown that the field effect is still present above $2 \mathrm{~K}$ and disappears only near 12-15 $\mathrm{K}$. As the value $T_{\mathrm{g}}=1.8 \mathrm{~K}$ has been obtained at very low frequency, these results are to be related to the variation of $T_{\mathrm{g}}$ with frequency [9]. The most important phonons in the heat current spectrum have a frequency $v_{\max }$ such that $h v_{\max } \sim 3.8 k T$, that is $v_{\max } \simeq 160 \mathrm{GHz}$ for $T=2 \mathrm{~K}$, and the corresponding freezing temperature calculated using the Fulcher law proposed by Holtzberg et al. [9] is $4.6 \mathrm{~K}$. Therefore it is not surprising that the spins appear to be frozen at $2 \mathrm{~K}$ in the thermal conductivity experiment. We note that, in the case of amorphous spin glass like the metallic alloys studied recently [10], the thermal conductivity again does not display any detectable anomaly at $T_{\mathrm{g}}$, but an increase of the phonon component with applied magnetic field is observed only in one case. These systems are in fact much more complicated than $\mathrm{Eu}_{x} \mathrm{Sr}_{1-x} \mathrm{~S}$ as they are metallic and both structurally and spin disordered.

A simple quantitative analysis of the results below $1 \mathrm{~K}$ has been carried out within the Debye model, where the thermal conductivity is given by [11] :

$$
K=\frac{k}{2 \pi^{2} v}\left(\frac{k T}{\hbar}\right)^{3} \int_{0}^{\theta / T} \frac{x^{4} \mathrm{e}^{x}}{\left(\mathrm{e}^{x}-1\right)^{2}} \tau(x) \mathrm{d} x .
$$

(The symbols have their usual meaning and $x=\hbar \omega / k T$ ). We have used a total inverse relaxation time $\tau^{-1}=v / L+G \omega$ where the first term corresponds to boundary scattering. The value of the mean sound velocity, $v=2.9 \times 10^{5} \mathrm{~cm} \cdot \mathrm{s}^{-1}$ has been calculated from the values of longitudinal and transverse velocities measured on a sample with $x=0.54$ [12]. The corresponding Debye temperature is $\theta=226 \mathrm{~K}$. 
Satisfactory fits to the experimental curves have been obtained and are illustrated in figure 2 . The values of the parameters are $v / L=1.1 \times 10^{6} \mathrm{~s}^{-1}, G_{0}=12.9 \times 10^{-5}(H=0)$ and $G_{H}=8 \times 10^{-5}(H=7$ teslas $)$. The calculated value of $v / L$ obtained from the dimensions of the sample is $2.9 \times 10^{6} \mathrm{~s}^{-1}$. The agreement is reasonable in view of the uncertainty on the sound velocity, and the crudeness of the model, where phonon focussing and specular reflexion are not taken into account.

Now the inverse relaxation time corresponding to phonon scattering by TLS is given by [8] :

$$
\tau_{\mathrm{TLS}}^{-1}=\frac{\pi n_{0} \bar{M}^{2}}{\rho v^{2}} \omega \tanh (\hbar \omega / 2 k T) .
$$

As already noted, the frequency of the most important phonons is such that $\hbar \omega_{\max } / k T \simeq 4$ so that the hyperbolic tangent can be neglected and the value of $n_{0} \bar{M}^{2}$ deduced from the coefficient $G$. The part of the scattering due to magnetic disorder corresponds to the difference $G_{0}-G_{H}$, as the saturation of the field effect is obtained. From the experimental value $G_{0}-G_{H}=4.9 \times 10^{-5}$, we obtain $n_{0} \bar{M}^{2}=5.8 \times 10^{6} \mathrm{erg} . \mathrm{cm}^{-3}$ (using the measured value of $\rho=4.4$ ). This value of $n_{0} \bar{M}^{2}$ is much smaller than found in structural glasses $\left(9 \times 10^{7} \mathrm{erg} . \mathrm{cm}^{-3}\right.$ in vitreous silica [13] $)$ and these "TLS " have not been detected by ultrasonic measurements [12]. The situation is similar to that observed in electron irradiated quartz [14]. A very crude estimation of the density of states $n_{0}$ can be made from the linear term of the specific heat measured by Meschede et al. [6] on a sample with $x=0.4$, and gives a large value $n_{0}=1.6 \times 10^{37} \mathrm{erg}^{-1} . \mathrm{cm}^{-3}$ which would lead to a very low coupling constant $\bar{M} \simeq 4 \times 10^{-4} \mathrm{eV}$. This number is indeed very speculative, but it should be noted that the isolated $\mathrm{Kramers}$ ion $\mathrm{Eu}^{2+}$ has an orbital singlet ground state ${ }^{8} \mathrm{~S}_{7 / 2}$ so that its coupling to the lattice is probably quite small.

It seems unlikely that the frequency dependent phonon scattering still present in high magnetic field would be magnetic in origin. This scattering, which is also described by a $G \omega$ term in the inverse relaxation time, could be due to isolated dislocations. In that case, the expression of $G$ is given by [11] :

$$
G \simeq \text { const. } \times N_{\mathrm{d}} b^{2} \gamma^{2}
$$

where $b$ is the Bürger vector of the dislocation, $\gamma$ the Grüneisen constant and the constant of the order of $10^{-1}$. The density of dislocations $N_{\mathrm{d}}$ corresponding to $G_{H}=8 \times 10^{-5}$ should be of the order of : $2 \times 10^{11} \mathrm{~cm}^{-2}$. Despite the fact that the above expression overestimates often $N_{\mathrm{d}}$ by a factor of $10^{2}$ or $10^{3}$, this value appears too large to be physically meaningful. This scattering could perhaps be related to some inhomogeneity of the distribution of the Eu ions, which has been observed in some $\mathrm{Eu}_{x} \mathrm{Sr}_{1-x} \mathrm{~S}$ crystals [15]. Two types of effects could occur : (i) some regions having different density and sound velocities than the mean crystal act as phonon scatterers; such a process leads to an apparent $G \omega^{p}$ term, with $p \simeq 1$, if there is a distribution of dimensions of the clusters [16]; (ii) in the calculation of the point defect scattering term related mainly to the mass difference between the Eu and $\mathrm{Sr}$ atoms, interference effects cannot be neglected and could lead to a frequency dependence different from the standard expression $A \omega^{4}$ [17].

The most interesting result of the present work is the evidence of phonon scattering by the magnetic disorder of the spin glass state. In the phenomenological analysis given above, we have considered the existence of «TLS », that is of localized entities. In fact the specific heat data in zero field [6] has been semi-quantitatively explained by computer simulation calculations of the density of states of collective excitations more analogous to spin waves [18, 19]. A contribution to the heat conductivity due to spin waves and varying as $T^{2}$ has been observed in a ferromagnetic EuS polycrystalline sample [20]. Such a possibility is excluded in $\mathrm{Eu}_{0.44} \mathrm{Sr}_{0.56} \mathrm{~S}$ as the effect of a magnetic field should be to decrease this contribution, in contrast with the experimental observation of an increase of the thermal conductivity. 
On the other hand, it is to be noted that a phenomenological description of the time dependent effects in the magnetic properties of metallic spin glasses has been given on the basis of thermally activated two level systems [21]. The possibility of tunnelling has not been considered in this work, though this process is important in the models describing the low temperature properties of structural glasses [7]. From the estimate of the density of states $n_{0}$ given above, $n_{0}=1.6 \times 10^{37} \mathrm{erg}^{-1}$ $\mathrm{cm}^{-3}$, and with the hypothesis that this constant density of states extends to energies of the order of $1 \mathrm{~K}$, the total number of «TLS » is found to be $2.2 \times 10^{21} \mathrm{~cm}^{-3}$, that is about one third of the number of Eu atoms. Therefore it appears that such "TLS » would correspond to small clusters and so are not the same as those responsible for the time-dependent effects, which imply the association of a large number of individual moments [21].

In fact, no memory effect has been detected in the thermal conductivity experiments described above and the results obtained above $1.2 \mathrm{~K}$ in a preliminary experiment were independent of the temperature of application of the magnetic field $(4.2$ or $1.2 \mathrm{~K})$. Our experimental conditions below $1 \mathrm{~K}$ (time scale of one measurement of the order of one hour at the lowest temperature, necessary to reach thermal equilibrium, and large magnetic field) are actually completely different from those of the study of magnetic properties by Ferré et al. [22].

Finally, we note that the theoretical studies of sound attenuation in spin glasses, which lead to an inverse relaxation time proportional to $\omega^{3 / 2}$ have been restricted so far to the temperature region around and above $T_{\mathrm{g}}[23,24]$. The results of the present work show that this frequency dependence is effectively not valid below $T_{\mathrm{g}}$.

4. Conclusion. - Measurements of the thermal conductivity of an $\mathrm{Eu}_{0.44} \mathrm{Sr}_{0.56} \mathrm{~S}$ single crystal with applied magnetic field have shown the evidence of phonon scattering by some magnetic entities related to the spin disorder of the spin glass phase $\left(T \ll T_{\mathrm{g}}\right)$. The $T^{2}$ law observed at low temperatures strongly suggests that these magnetic entities scatter phonons in a similar way to the TLS involved in the properties of structural glasses. Such a phenomenological analysis of the results of both specific heat data [6] and thermal conductivity leads to the conclusion that the magnetic « TLS » should be different from those invoked by Prejean and Souletie [21], where only thermally activation processes are operative. Nevertheless this point must be confirmed by (i) specific heat measurements on the sample used in the present work, in high magnetic fields, to obtain a more reliable quantitative estimate of the density of TLS, (ii) magnetization measurements, again on the same sample, in the same magnetic field and temperature ranges as used here, to control any time dependence and memory effects. Theoretical work on the microscopic nature of these magnetic « TLS » and their coupling to phonons should be of great interest. Finally, the phonon scattering by magnetic « TLS » disappears above 2 teslas at $T=0.288 \mathrm{~K}$, and the question arises as to the possible relation between this « saturating » field and the predicted transition line in the $(H, T)$ plane between the spin glass and paramagnetic phases [25, 26]. Additional experiments with applied magnetic field at different temperatures are clearly needed and will be carried out in a near future.

Acknowledgments. - We are very grateful to R. Orbach and J. L. Tholence for useful discussions, R. Maynard for critical reading of the manuscript and helpful suggestions, and T. Baumann for communication of results prior to publication. We want to thank Mr. Arnaud and Mr. Favre for their technical assistance during the measurements. 


\section{References}

[1] Maletta, H. and Felsch, W., Phys. Rev. B 20 (1979) 1245.

Holtzberg, F., Tholence, J. L., Godfrin, H. and Tournier, R., J. Appl. Phys. 50 (3) (1979) 1717.

[2] RaJChenbaCh, J., Thèse de 3e cycle, Orsay (1980) (unpublished).

[3] Maletta, H. and Felsch, W., Z. Phys. B 37 (1980) 55.

[4] Toulouse, G., Comm. Phys. 2 (1977) 115.

[5] Deville, A., Arzoumanian, C., Gaillard, B., Blanchard, C., Jamet, J. P. and Maletta, H., $J$. Physique 42 (1981) 1641.

[6] Meschede, D., Steglich, F., Felsch, W., Maletta, H. and Zinn, W., Phys. Rev. Lett. 44 (1980) 102.

[7] Anderson, P. W., Halperin, B. I. and Varma, C. M., Philos. Mag. 25 (1972) 1.

[8] See for instance Hunklinger, S. and Arnold, W., in Physical Acoustics 12, eds Mason W. P. and Thurston R. N. (Academic Press, New York) 1976 p. 155.

[9] Holtzberg, F., Francavilla, T. L., Huang, C. Y. and Tholence, J. L., J. Appl. Phys. 53 (3) (1982) 2229.

[10] Herlach, D. M., Wassermann, E. F. and Kästner, J., Phys. Rev. B 24 (1981) 3204.

Herlach, D. M., Wassermann, E. F. and WillneCker, R., I.C.M. Conf. Kyoto (1982).

[11] See for example Berman, R., Thermal conduction in Solids (Clarendon) 1976.

[12] BaumanN, Th., private communication.

[13] Golding, B., Graebner, J. E., Schutz, R. J., Phys. Rev. B 14 (1976) 1660.

[14] Laermans, C., De Goër, A. M. and Locatelli, M., Phys. Lett. 80A (1981) 331.

[15] Hüser, D., Daub, G., Gronau, M., Methfessel, S. and Wagner D., J. Magn. Magn. Mater. 15-18 (1980) 207.

[16] Seyfert, P., J. Low Temp. Phys. 2 (1970) 555.

[17] KLemens, P. G., Solid State Phys. 7 (1958), Ed. F. Seitz and D. Turnbull, p. 1.

[18] Ching, W. Y., Huber, D. L. and Leung, K. M., Phys. Rev. B 21 (1980) 3708.

[19] KREY, U., Z. Physik B 38 (1980) 243.

[20] Mc Collum, D. C., Wild, R. L. and Callaway, J., Phys. Rev. 136 (1964) A 426.

[21] Prejean, J. J., Souletie, J., J. Physique 41 (1980) 1335.

[22] Ferre, J., Rajchenbach, J. and Maletta, H., J. Appl. Phys. 52 (1981) 1697.

[23] Fischer, K. H., Z. Physik. B 43 (1981) 291.

[24] Hertz, J. A. and Khurana, A., Phys. Rev. Lett. 46 (1981) 496 ; Khurana, A., Phys. Rev. B 25 (1982) 452.

[25] De Almeida, J. R. L. and Thouless, D. J., J. Phys. A 11 (1978) 983.

[26] Toulouse, G., J. Physique Lett. 41 (1980) L-447.

Toulouse, G., Gabay, M., J. Physique Lett. 42 (1981) L-103. 